\title{
Accurate, Swift and Noiseless Image Binarization
}

\author{
Wassim Al-Khawand ${ }^{1}$, Seifedine Kadry ${ }^{2, *}$, Riccardo Bozzo $^{3}$, Khaled Smaili ${ }^{4}$ \\ ${ }^{1}$ School of Engineering Sciences and Technologies University of Genoa - UNIGE, Genoa, Italy. \\ ${ }^{2}$ School of Engineering American University of the Middle East, Kuwait. \\ ${ }^{3}$ DITEN-Dept. of Electrical, Electronic, Telecommunications Engineering and Naval Architecture University of Genoa, Genoa, Italy. \\ ${ }^{4}$ Faculty of Sciences, Lebanese University, Lebanon.
}

(Received: 28 March 2015; Accepted: 18 October 2015)

\begin{abstract}
In this paper, we present an accurate, swift and noiseless image binarization technique that was tested on real life back side container images. Our approach consists of transferring a colored image into grayscale, then to construct the histogram which will be divided into group of colors and after that, the foreground -that can be darker or lighter than the background- will be automatically identified and finally, the foreground boundaries will be assiduously enhanced before creating the binarized image. The average processing time of our proposed method is less than 8 milliseconds which makes it highly suitable for real life multi-user applications.
\end{abstract}

Keywords Binarized Image, Histogram, Automatic Foreground Detection, Container Number

AMS 2010 subject classifications 94A08, 68U10

DOI: $10.19139 /$ soic.v4i1.140

\section{Introduction}

Image binarization is usually performed in the preprocessing stage of image processing; because binarization is one of the main phases of the Optical Character Recognition (OCR) and consists of transforming a grayscale image (i.e., image of up to 256 gray levels) into a binary image (i.e., black and white image), our proposed approach will focus on the foreground extraction (i.e., useful information part of an image) in order to be used by an OCR application.

\section{Related Works}

Due to the high importance of binarization, plenty of researches were published over the past four decades; to this end, we will briefly summarize some of the recent ones. Chaki et al. [1] introduced a detailed survey about the principles of image binarization techniques. Gill [2] evaluated the different image binarization techniques to find the gaps in existing ones. Manju and Jijina [3] proposed a technique that makes use of the adaptive image contrast and some of the noise reduction methods. Kaur and Mahajan [4] integrated the image gradients and the image contrast enhancement to improve the accuracy of document image binarization and they also utilized the guided image filter to further improve the accuracy rate. In the proposed method of Chaudhary and Saini [5], an image having high contrast is constructed then a hybrid algorithm for thresholding -global and local thresholding

\footnotetext{
${ }^{*}$ Correspondence to: Seifedine Kadry (Email: skadry@gmail.com). School of Engineering American University of the Middle East,
} Kuwait.

ISSN 2310-5070 (online) ISSN 2311-004X (print)

Copyright (C) 2016 International Academic Press 
methods- where the global thresholding step has been modified such that the output will not be a binarized image but an intermediate gray level image and finally the local thresholding will be applied. Shukla et al. [6]presented an approach for enhancing degraded documents images using binarization technique that consists of an adaptive image contrast document image binarization technique where the image is constructed using local image gradient and local image contrast, then further edge estimation algorithm is used to identify the text stroke edge pixels and finally the text within the document is segmented by a thresholding technique which is based on the height and width of letter size. Pratikakis et al. [7] presented in the DIBCO 2013 their paper that includes a short description of 23 submitted methods. Ntirogiannis et al. [8] addressed a pixel-based binarization evaluation methodology for historical handwritten/machine-printed document images. Su et al. [9] proposed an adaptive contrast map which is constructed for an input degraded document image, then the contrast map is binarized and combined with Cannys edge map to identify the text stroke edge pixels and finally the document text is segmented by a local threshold that is estimated based on the intensities of the detected text stroke edge pixels within a local window. Su et al. [10] proposed a method to segment text from the background region of a document image and a self-learning classification framework that combines binary outputs of different binarization methods where their framework uses the sparse representation to re-classify the document pixels in order to generate better binary results. Wagdy et al. [11] proposed a document image clean up and binarization method based on retinex theory and global threshold. Vincent et al. [12] proposed an approach to expect the result of binarization algorithms on a given document image according to its state of degradation by using different features based on the intensity, quantity and location of the degradation in order to build prediction models of binarization algorithms which will be used to pick the best binarization algorithm for a given document image. Minenobu et al. [13] proposed a binarization method based on the line and character areas that are estimated via the form structure analysis and by subtracting the background then, the color shift is detached by using morphological processing and finally each pixel of the subtracted background image is discriminated into character strings and lines by using dynamic color classification. Abdenour et al. [14] presented a scheme for binarization of ancient and degraded document images, grounded on texture qualities by suggesting an adaptive threshold-based technique. Ziaei et al. [15] presented an unsupervised post processing technique founded on the phase-preserved denoised image and also phase congruency features extracted from the input image where the main part of their technique comprised two mask images that can be used to cross the false positive pixels on the production of their binarization technique. Jon et al. [16] described an automated image enhancement and binarization approach that doesnt require data training and which can be applied to images of typewritten text as well as hand written text or a mixture of both. Vassilis et al. [17] proposed a method based on mathematical morphology for extracting text regions from degraded handwritten document images where they started by a top-hat-by-reconstruction to produce a filtered image with reasonable even background then by region growing, starting from a set of seed points while attaching to each seed similar intensity neighbouring pixels and finally a conditional extension of the initially detected text regions based on the values of the second derivative of the filtered image is applied. Su et al. [18] proposed a learning framework that makes use of the Markov Random Field to advance the performance of the existing document image binarization methods for the degraded document images. Patvardhan et al. [19] proposed a binarization method of document images suitable for OCR via discrete curvelet transform in order to eliminate difficult image background and white Gaussian noise. Sokratis et al. [20] presented a hybrid approach that starts by applying a global thresholding technique then by identifying the image areas that are more likely to still contain noise and finally each of these areas is re-processed separately to achieve better binarization quality. Zhang and $\mathrm{Wu}$ [21] used wiener filter to reduce noises then applied an improved adaptive Otsus method for binarization and finally dilation and erosion operators are used to preserve stroke connectivity and to fill possible breaks, gaps, holes where packed binary format and destination word accumulation are also put in place to hasten the dilation and erosion processing. Su et al. [22] proposed a classification framework to combine different thresholding methods in order to produce a better performance for document image binarization then, given the binarization results of some reported methods, the proposed framework divided the document image pixels into three sets, namely foreground pixels, background pixels and uncertain pixels and finally, a classifier is applied to iteratively classify the uncertain pixels into foreground and background. Shaikh et al. [23] proposed an iterative partitioning for logically segmenting an image into four rectangular parts until a certain criteria is satisfied 
then a threshold value is found for each partition using Otsus method and finally, a global threshold is calculated by taking the mean of the threshold values for all the partitions.

\section{Context Overview}

Due to the high importance of containers in the shipping field, and in order to facilitate and accelerate trade by automatically recognizing the container number (e.g. Customs gates, terminal operators gates, quays, ), and after presenting new techniques applied on container images for reading skewed images without image rotation [24] and for skew estimation [25], we present hereafter our proposed approach for one of the crucial phases of image binarization that we tested and returned excellent results on container images. Fig. 1 illustrates an image taken for the back side of a container.

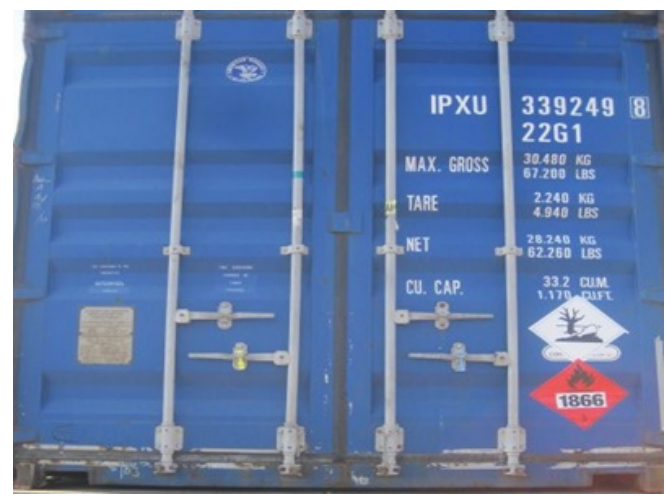

Figure 1. Back Side Container Image.

As shown in Fig. 1, the most important information that exists on a container is its number which is written on the upper right line of the above figure; according to ISO 6346, the container has the following structure: Owner code (3 digits), category identifier (1 digit), serial number (6 digits) and a check digit (1 digit); for example in Fig. 1, the owner code is IPX, the category identifier is U, the serial number is 339249 and the check digit is 8 . To binarize such an image subject to an OCR system, it will be enough to binarize its upper right part; to this end and to accelerate the whole process, we will confine our work to the interesting part of such images noting that the same algorithm can be applied for the whole image. Fig. 2 illustrates the interesting part of the back side container image.

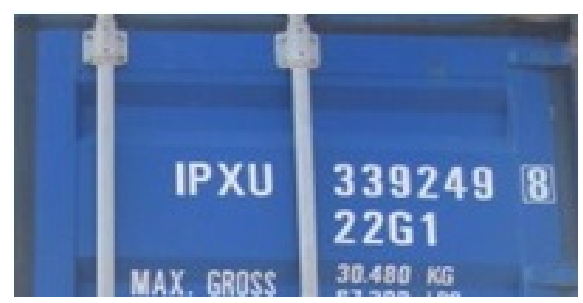

Figure 2. Interesting part of the back side container image.

\section{Challenges}

Most of the published binarization techniques focus on document image binarization where the foreground is darker than the background (e.g., [2, 22]) while our proposed method deals with images (and can also be applied 
to document images) taken from real-life scenarios where the foreground can be darker (Fig. 3) or lighter (Fig. 4) than the background.

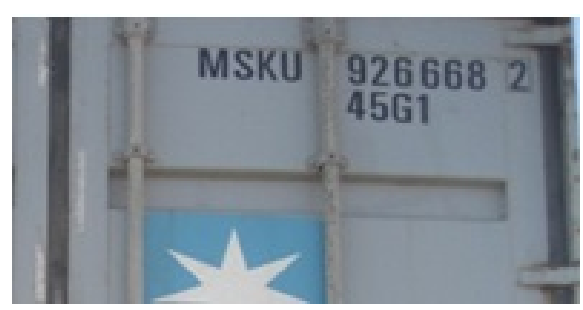

Figure 3. Foreground darker than background.

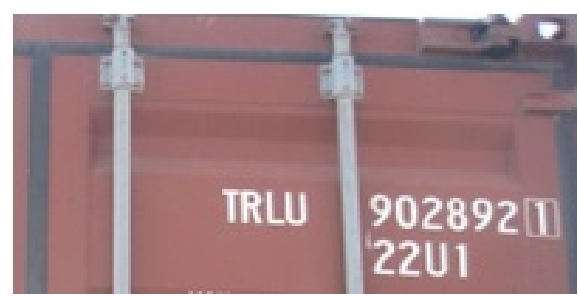

Figure 4. Foreground lighter than background.

Worthy to note that real-life images present an infinite number of cases where each one can be a challenge by itself and thus, due to the diversity and unpredictable nature of such an environment, binarization becomes much more interesting; for example, fluid leakage (fig. 5), existence of objects irrelevant to the image (fig. 6), different shadow volume and intensity (fig. 7), different type and quantity of mud and dust (fig. 8), fog, rain, uneven or insufficient illumination, smears, faint characters, ..., or a combination of them (fig. 9).

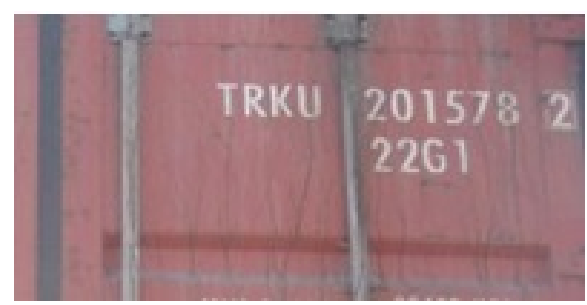

Figure 5. Fluid leakage.

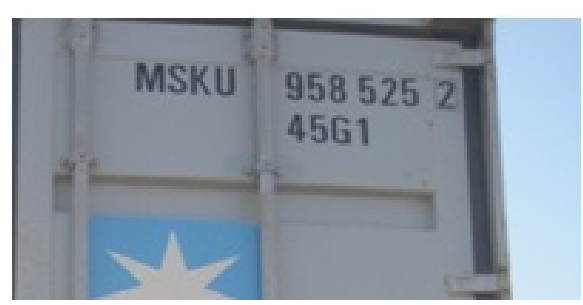

Figure 6. Sky inside a container image. 

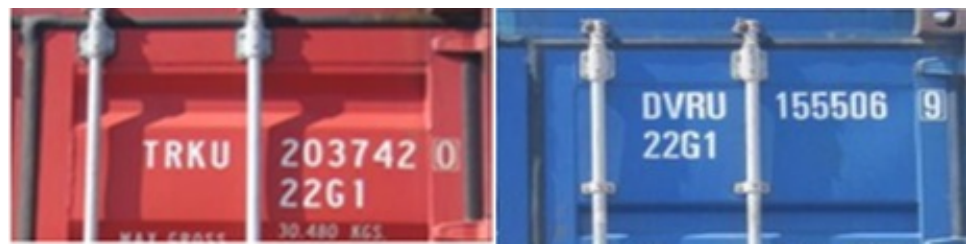

Figure 7. Different shadow volume and intensity.

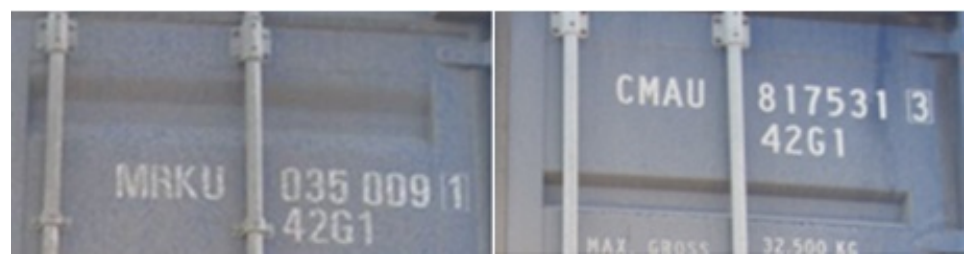

Figure 8. Different type and quantity of mud and dust.

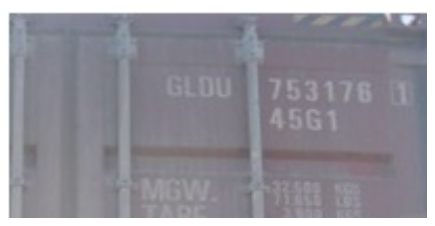

Figure 9. Leakage, mud, fog, ...

\section{Proposed Method}

After acquiring the interesting part of the colored image and converting it to a grayscale image, our proposed method consists of the following phases:

5.1 Histogram Creation;

5.2 Histogram Division;

5.3 Foreground Identification;

5.4 Foreground Boundaries Pinpointing;

5.5 Binary Image Creation.

\subsection{Histogram Creation}

This phase consists of filling a vector of dimension 256 with the number of occurrences of each grayscale value that exists in the grayscale image (the index of the vector goes from 0Cwhich is black- to 255 Cwhich is white-). Fig. 10 illustrates a graphical representation of such an histogram.

N.B.: Worthy to note that while moving from the left to the right side of an histogram (i.e., from lower to higher index), we will be moving from dark to light colors.

\subsection{Histogram Division}

This phase consists of smoothing the histogram then dividing it into groups of colors (i.e., each hill in the histogram represents one group of colors). Fig. 11 illustrates an histogram which corresponds to a grayscale image having 4 different groups of colors.

N.B.: 1) In order to accelerate the processing time, for each histogram group a set of properties is created, among them are the lower and upper limit (i.e., index) of each group. 2) The length of a group is its number of indexes. 


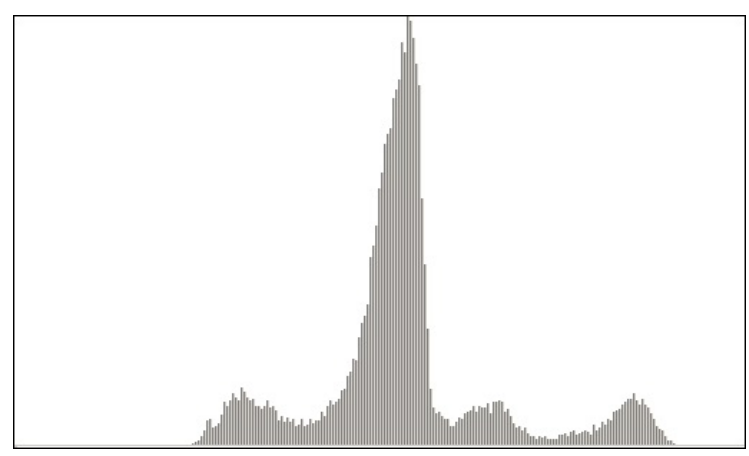

Figure 10. An histogram example.

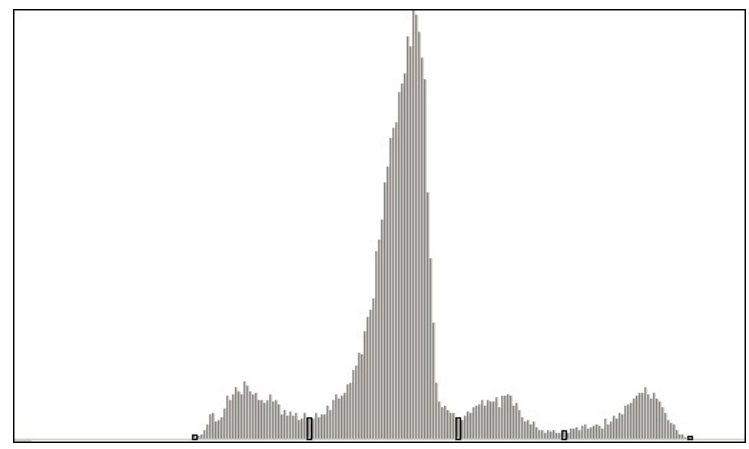

Figure 11. A divided histogram.

\subsection{Foreground Identification}

Our proposed algorithm is able to differentiate the foreground (useful part of the image) from the remaining parts of the image and avoids any human intervention for specifying whether the image has a foreground darker than background or vice versa; this phase consists of:

$\mathrm{C} 1$. Determination of the background -represented hereafter by BACK- as being the histogram group having the highest peak while taking into consideration the number of grayscale pixels and the BACK length.

C2. Division of the histogram into two partitions where the first one consists of the histogram groups that exist on the left side of BACK -represented hereafter by LEFT- and the second one consists of the histogram groups that exist on the right side of BACK -represented hereafter by RIGHT- then, the number of groups in each partition along with some of their properties (e.g., the number of grayscale pixels and the length of each partition) are weighted and the partition having the highest score will be the winner Crepresented hereafter by WIN-. For example in the fig. 11, RIGHT wins and thus, the foreground is lighter than the background.

C3. Because the foreground color should be far from the background color in order to be easily read, we fix the foreground as being the farthest group of WIN relatively to BACK.

\section{N.B.:}

1) Among the numerous colors that may exist in an image (e.g., Fig. 6 contains the colors: black, light blue, blue, gray and white), our proposed approach targets the useful group of colors in the image (i.e., the part that will be used in an OCR application and which is the foreground).

2) In order to surpass the problem related to the strong (or weak) illumination gradient, our approach disregards the extremity group of colors in the histogram in case they are disconnected from the other groups of colors (e.g., the existence of the sky, sun, inside the container image).

3) In our approach, all the histogram values less than 15 are disregarded. 


\subsection{Foreground Boundaries Pinpointing}

In order to take into consideration the small divergence that exists between theory and real-life scenarios (i.e., due to fluid leakage, muds, shadows, ) our approach applies a small modification to the foreground border which may increase the upper limit (if LEFT wins) or decrease the lower limit (if RIGHT wins) in a way that the foreground can make use of some colors from its adjacent group(s) of colors; to be more precise:

1. The length of the foreground should be at least $5 \%$ of the histogram effective length.

2. The foreground number of pixels should be at least $5 \%$ of the image total number of pixels.

3. In all cases, the foreground should not make use of the background by more than $1 / 6$ of the background length. Fig. 12 illustrates the case where the useful check digit number (i.e., rightmost number of the first line) has a gray color while the foreground is white which clarifies why some colors should be borrowed from the group adjacent to the foreground.

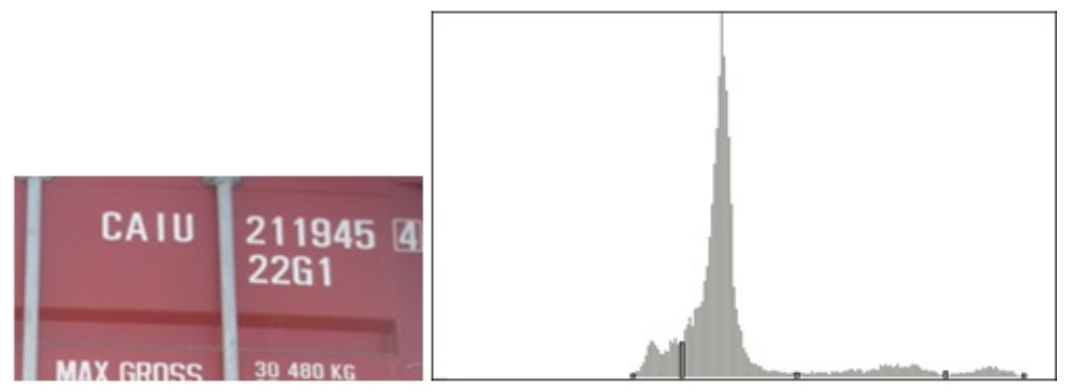

Figure 12. Fade check digit character.

\subsection{Binary Image Creation}

Having specified the borders of the foreground (i.e., lower and upper histogram limits), a white image is created where only the part of the grayscale image that corresponds to the foreground is written in black.

\section{Proposed Method Flowchart}

Fig. 13 illustrates the general flowchart of our proposed method.

\section{Experiments}

Our proposed method was tested to binarize grayscaled back side container images and it succeeded to return very good results regarding its accuracy, performance and noiseless results; our approach was tested on 400 manually taken container images randomly selected among 50 different companies; The result of our experiments returned an average response time less than 8 milliseconds while using a laptop having a $2.00 \mathrm{GHz}$ processor and $2 \mathrm{~GB}$ of RAM, which makes our proposed method very convenient and highly desirable for real-time applications. Even though our proposed method implicitly filters the binary image, it has the option to run for OCR applications and thus, the result image will contain much less noise and the useless box around the container check digit will be alleviated, or most of the times, removed. We will illustrate in the Fig. 14, 15, 16, 17 and 18 some samples from our experimental results. To make the figures more readable, each figure will contain two rows where the upper one represents the colored image (on the left) and the grayscale image (on the right) where the lower row contains our default image binarization (on the left) and our image binarization for OCR applications (on the right). 

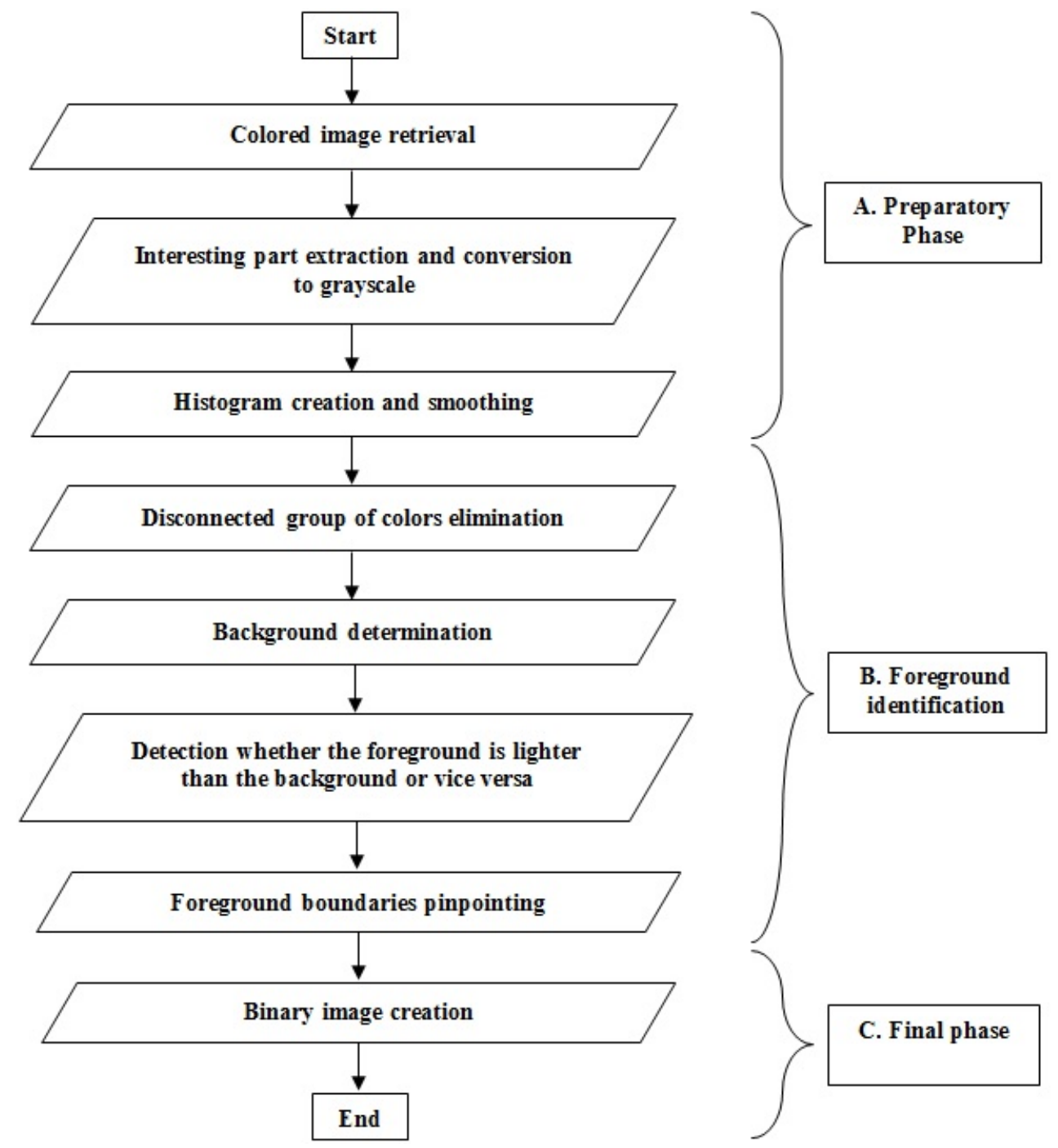

Figure 13. General flowchart of our proposed method.

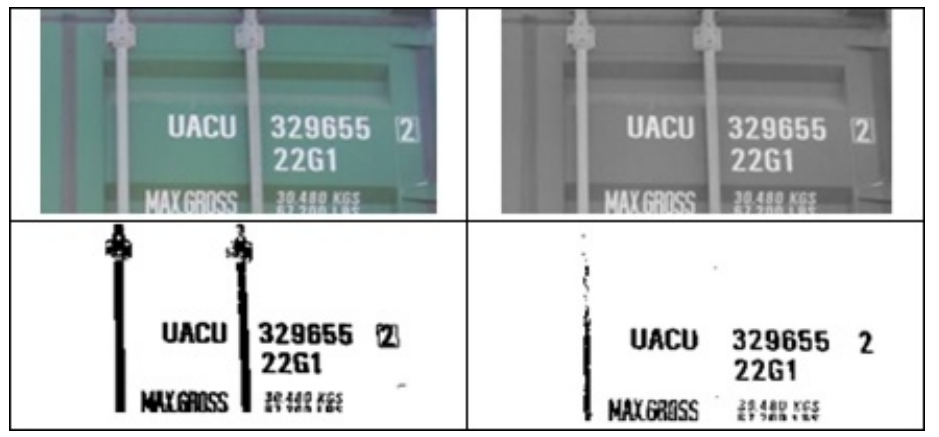

Figure 14. Green (background), white (foreground), black and gray colors. 


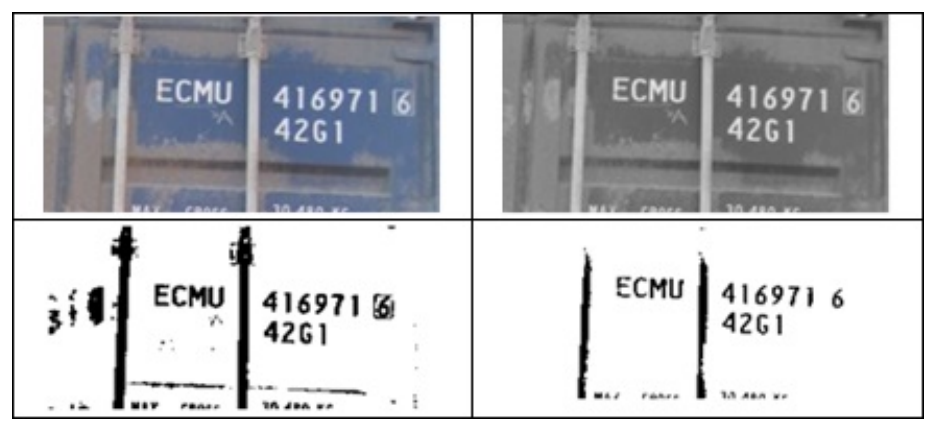

Figure 15. Image with dust.

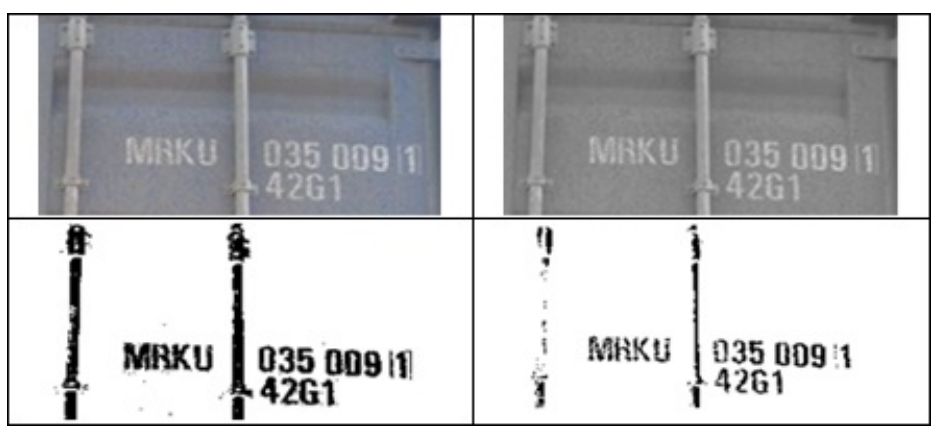

Figure 16. Image with mud.

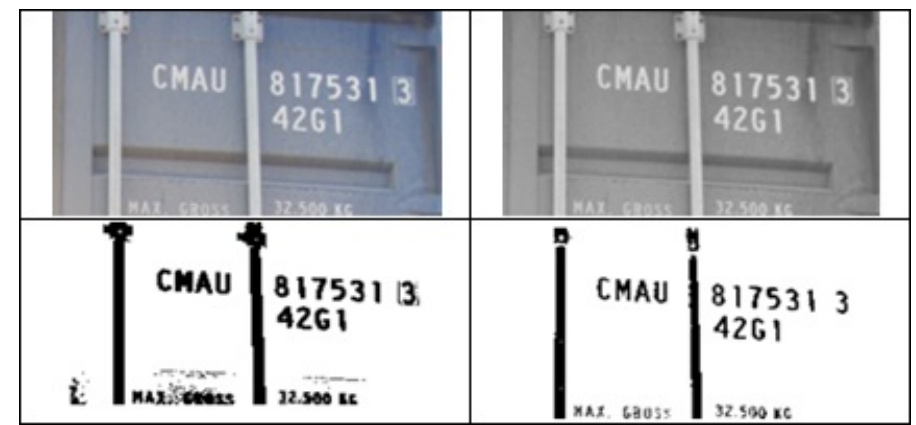

Figure 17. Image with degradated intensity.

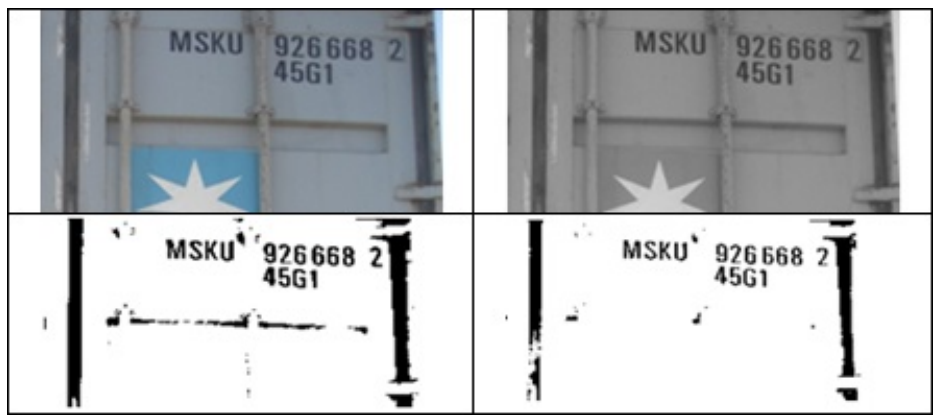

Figure 18. Image with a foreground darker than background. 


\section{Application Field}

Although this paper was intended to binarize the back side of a container image, but it can also be implemented to binarize any other type of images (e.g. Container owner name CFig. 19- and vehicle plate CFig. 20-) or handwritten or machine printed documents or a mixture of both.

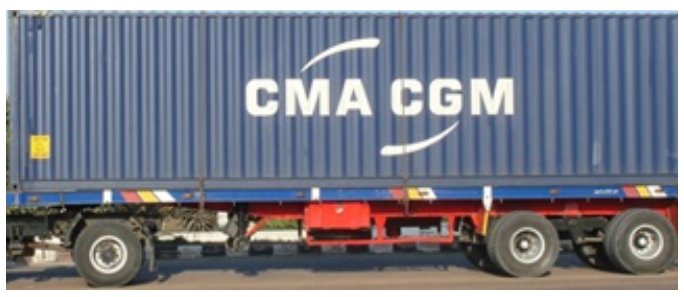

Figure 19. Container owner name.

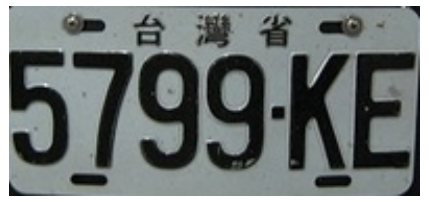

Figure 20. Vehicule plate.

\section{Comparative Study}

On the other hand and compared to most of the currently existing global or local thresholding techniques, our approach returned much better binary images quality (i.e., more precision with much less noise) if compared to local thresholding techniques and better or similar binary images if compared to global thresholding techniques.

N.B.: 1) In this section, the global and local thresholding methods (except ours) were retrieved from ImageJ software. 2) We will illustrate in the Fig. 21, 22 and 23 some samples from our experiments. To make the figures more readable, each figure contains three blocks where the upper one contains the colored image (on the left) the grayscale image (in the middle) and our proposed method (on the right), the middle block contains the local thresholding results (i.e., Bernsen, Contrast, Mean, Median, MidGrey, Niblack, Otsu, Phanslkar and Sauvola) and the lower block contains the global thresholding results (i.e., Huang, Intermodes, IsoData, Li, MaxEntropy, Mean, MinError, Minimum, Moments, Otsu, Percentile, RenyiEntropy, Shanbhag, Triangle and Yen).

To be objective in presenting our approach, we will introduce hereafter a table that contains a comparison between our proposed method and the other 21 methods; Figure 24 contains some statistics related to the result of using 400 randomly selected container images and it is worthy to note that the OCR that we used is freely accessible via the website http://www. onlineocr. net/.

N.B.: In Figure 24, the column Number of Recognized Container Digits is associated to the first 10 digits of a container number (i.e., the container check digit is excluded because it exists in a separate column).

N.B.: We dont claim that our approach is better than all the other techniques for all the images but our experiments has proved that most of the times our approach returned one of the best results and especially for images containing dust, mud, shadow, uneven illumination, fluid leakage, ... 


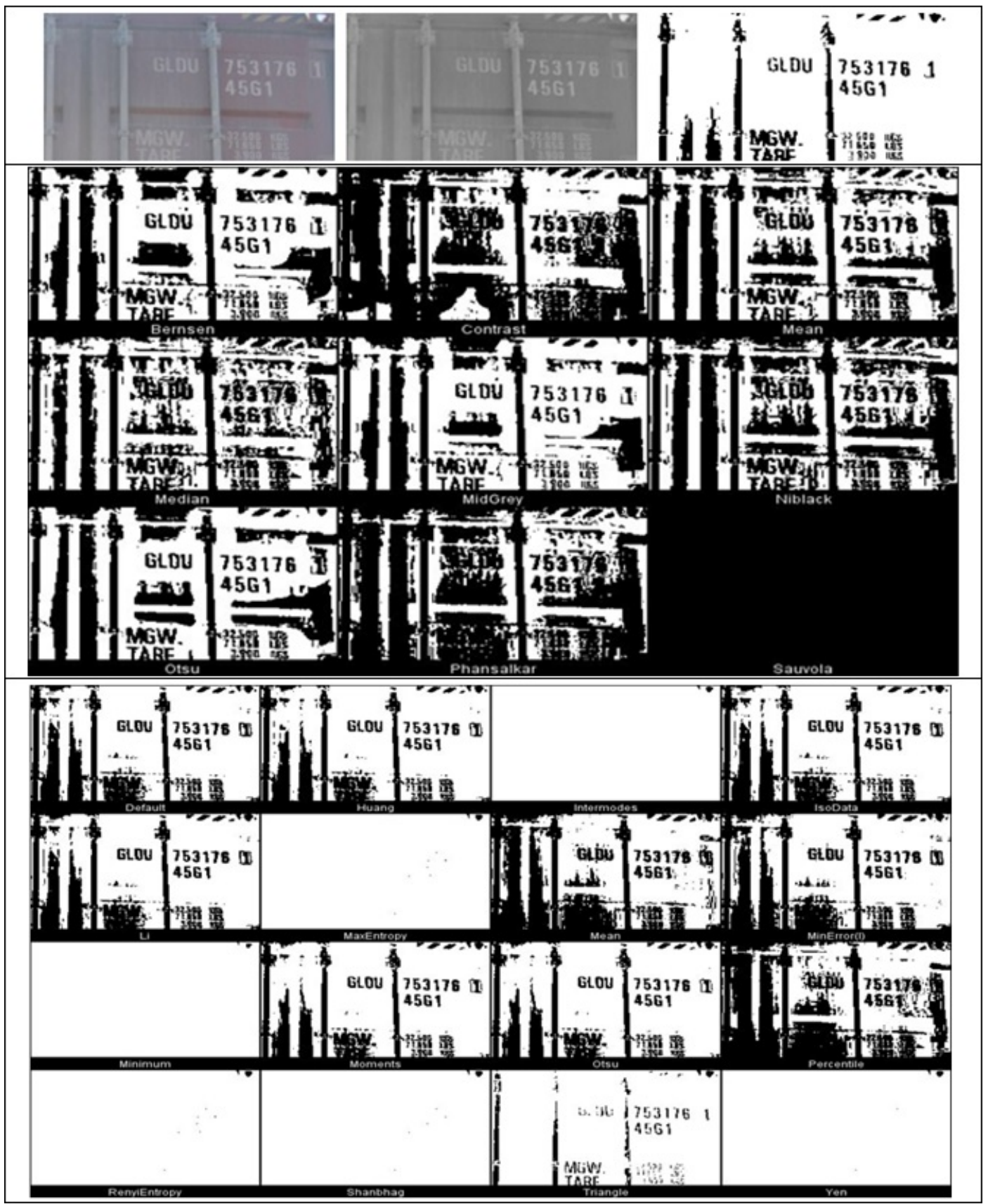

Figure 21. Image with mud, dust, uneven illumination... 


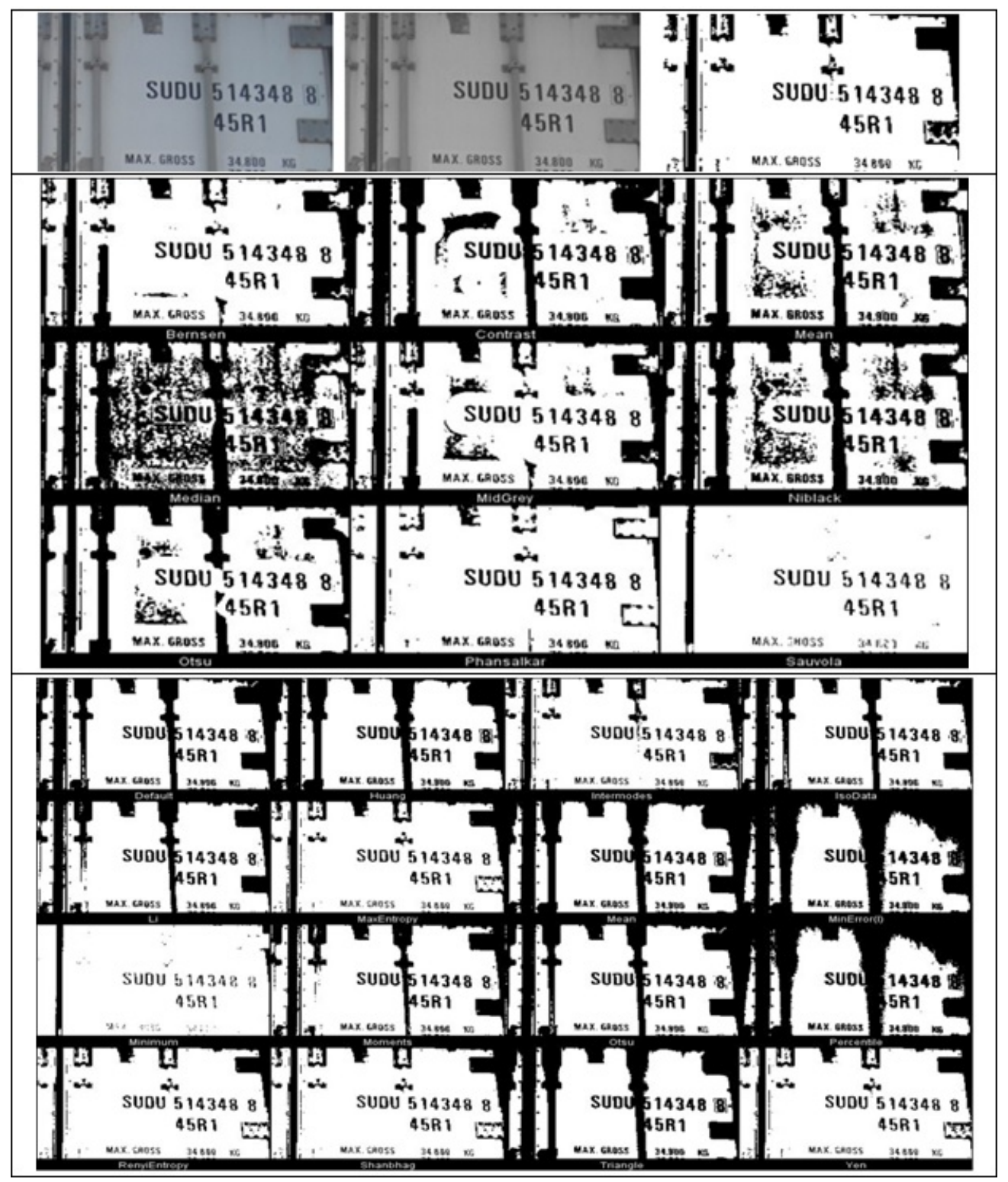

Figure 22. Image with a foreground darker than the background. 


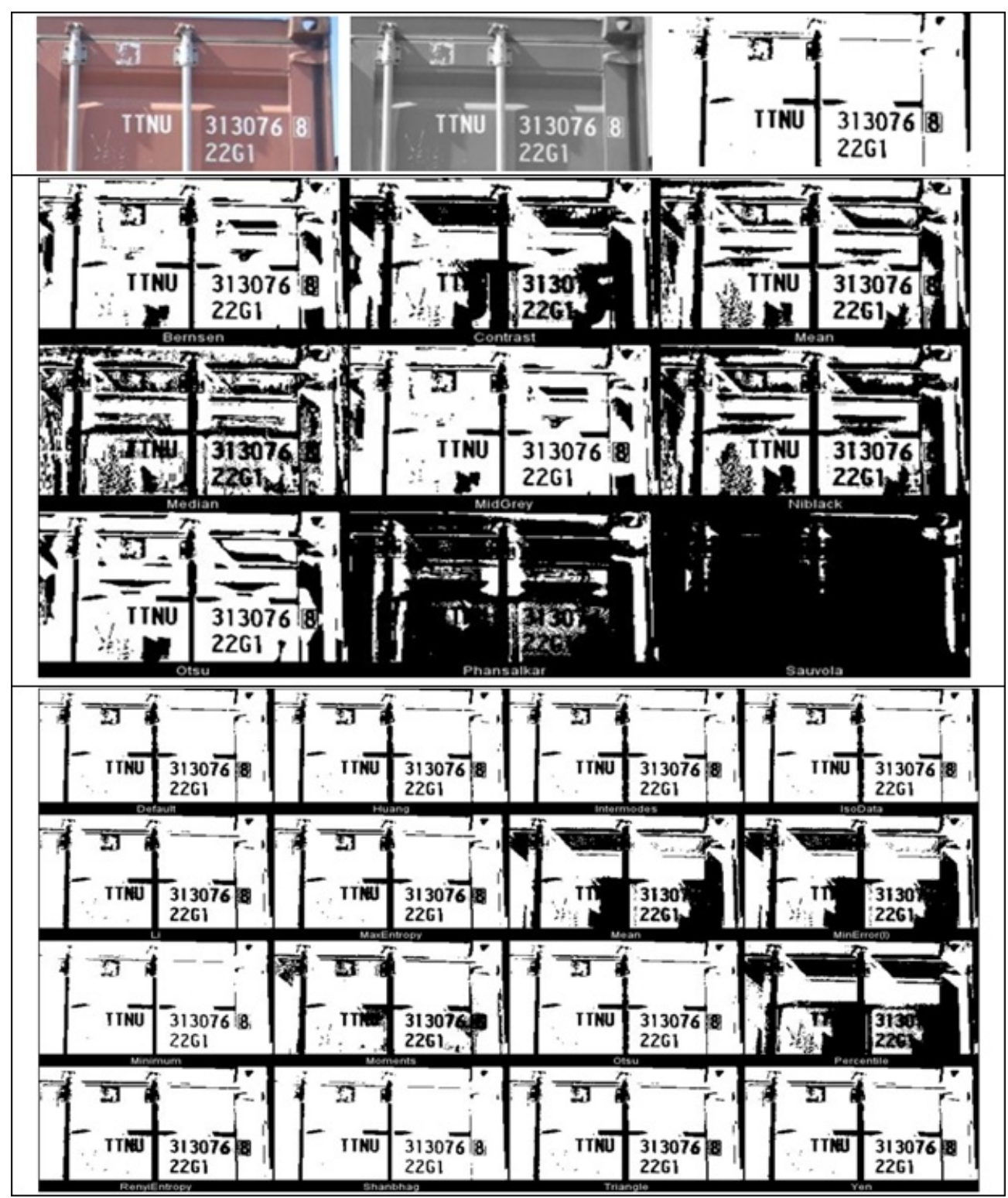

Figure 23. Image with shadow. 


\begin{tabular}{|c|c|c|c|c|c|c|c|}
\hline & Method & $\begin{array}{c}\text { Number of } \\
\text { Recognized } \\
\text { Images }\end{array}$ & $\begin{array}{c}\% \text { of } \\
\text { Recognized } \\
\text { Images }\end{array}$ & $\begin{array}{c}\text { Number of } \\
\text { Recognized } \\
\text { Container } \\
\text { Digits }\end{array}$ & $\begin{array}{c}\% \text { of } \\
\text { Recognized } \\
\text { Container } \\
\text { Digits }\end{array}$ & $\begin{array}{l}\text { Number of } \\
\text { Recognized } \\
\text { Container } \\
\text { Check Digits }\end{array}$ & $\begin{array}{c}\% \text { of } \\
\text { Recognized } \\
\text { Container } \\
\text { Check Digits }\end{array}$ \\
\hline \multirow{16}{*}{$\begin{array}{c}\text { Global } \\
\text { Thresholding }\end{array}$} & Our method & 400 & 100.00 & 3433 & 85.83 & 217 & 54.25 \\
\hline & Huang & 217 & 54.25 & 1472 & 36.80 & 80 & 20.00 \\
\hline & Intermodes & 208 & 52.00 & 1065 & 26.63 & 64 & 16.00 \\
\hline & IsoData & 288 & 72.00 & 2149 & 53.73 & 164 & 41.00 \\
\hline & $\mathrm{Li}$ & 217 & 54.25 & 1305 & 32.63 & 64 & 16.00 \\
\hline & MaxEntropy & 25 & 6.25 & 9 & 0.23 & 0 & 0.00 \\
\hline & Mean & 288 & 72.00 & 1961 & 49.03 & 144 & 36.00 \\
\hline & MinError & 185 & 46.25 & 1104 & 27.60 & 64 & 16.00 \\
\hline & Minimum & 297 & 74.25 & 2057 & 51.43 & 144 & 36.00 \\
\hline & Moments & 128 & 32.00 & 784 & 19.60 & 64 & 16.00 \\
\hline & Otsu & 80 & 20.00 & 320 & 8.00 & 9 & 2.25 \\
\hline & Percentile & 233 & 58.25 & 1376 & 34.40 & 128 & 32.00 \\
\hline & RenyiEntropy & 249 & 62.25 & 1609 & 40.23 & 137 & 34.25 \\
\hline & Shanbhag & 233 & 58.25 & 1568 & 39.20 & 128 & 32.00 \\
\hline & Triangle & 233 & 58.25 & 1881 & 47.03 & 112 & 28.00 \\
\hline & Yen & 217 & 54.25 & 1488 & 37.20 & 121 & 30.25 \\
\hline \multirow{6}{*}{$\begin{array}{c}\text { Local } \\
\text { Thresholding }\end{array}$} & Bernsen & 41 & 10.25 & 345 & 8.63 & 25 & 6.25 \\
\hline & Mean & 281 & 70.25 & 2137 & 53.43 & 153 & 38.25 \\
\hline & Median & 281 & 70.25 & 2144 & 53.60 & 153 & 38.25 \\
\hline & MidGrey & 121 & 30.25 & 144 & 3.60 & 9 & 2.25 \\
\hline & Niblack & 265 & 66.25 & 1913 & 47.83 & 112 & 28.00 \\
\hline & Sauvola & 281 & 70.25 & 2240 & 56.00 & 128 & 32.00 \\
\hline
\end{tabular}

Figure 24. An Objective Comparison.

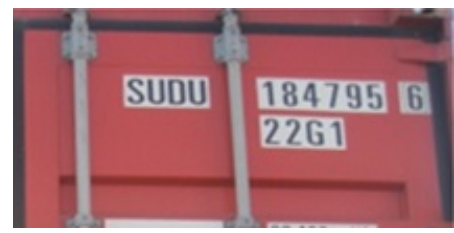

Figure 25. A black container number located a white rectangle inside a red container image.

\section{Future Works}

Even though our approach returned very good results and easily competes with all of the currently available binarization methods, it still need to be improved in order to cover the case where the container number has a dedicated background which is totally different from the container color; Fig. 25 illustrates such a case.

\section{REFERENCES}

1. N. Chaki et Al., A Comprehensive Survey on Image Binarization Techniques, Studies in Computational Intelligence 560, doi:10.1007/978-81-322-1907-1, Springer India, 2014.

2. Tarnjot Kaur Gill, Document Image Binarization Techniques- A Review, International Journal of Computer Applications (0975 C 8887), Volume 98C No.12, July 2014.

3. Manju Joseph and Jijina K.P., Simple and Efficient Document Image Binarization Technique For Degraded Document Images, IJSR - International Journal Of Scientific Research, Vol. 3. Issue 5, ISSN No. 2277 C 8179, May 2014.

4. Er. Jagroop Kaur and Dr.Rajiv Mahajan, Improved Degraded Document Image Binarization Using Guided Image Filter, IJSRE International Journal Of Scientific Research And Education, Vol. 2, Issue 7, Pages 1444-1452, ISSN (e): 2321-7545, July 2014. 
5. Prashali Chaudhary and B.S. Saini, An Effective And Robust Technique For The Binarization Of Degraded Document Images, IJRET - International Journal of Research in Engineering and Technology, Vol. 03, Issue 06, eISSN 2319-1163, pISSN 2321-7308, Jun 2014.

6. Sayali Shukla, Ashwini Sonawane, Vrushali Topale and Pooja Tiwari, Improving Degraded Document Images Using Binarization Technique, IJSTR C International Journal Of Scientific and Technology Research, Vol. 3, Issue 5, ISSN 2277-8616 333, May 2014.

7. Ioannis Pratikakis, Basilis Gatos and Konstantinos Ntirogiannis, ICDAR 2013 Document Image Binarization Contest (DIBCO 2013), 12th International Conference on Document Analysis and Recognition, 1520-5363, IEEE DOI 10.1109/ICDAR.2013.219, 2013.

8. Konstantinos Ntirogiannis, Basilis Gatos, and Ioannis Pratikakis, Performance Evaluation Methodology for Historical Document Image Binarization, IEEE Transactions On Image Processing, Vol. 22, No. 2, Page 595-609, February 2013.

9. Bolan Su, Shijian Lu, and Chew Lim Tan, Robust Document Image Binarization Technique for Degraded Document Images, IEEE Transactions On Image Processing, Vol. 22, No. 4, Page 1408-1417, April 2013.

10. Bolan Su, Shuangxuan Tian, Shijian Lu, and Thien Anh Dinh, Self Learning Classification for Degraded Document Images by Sparse Representation, 12th International Conference on Document Analysis and Recognition (ICDAR), IEEE, 2013.

11. Wagdy M., Faye Ibrahim, and Dayang Rohaya, Fast and efficient document image clean up and binarization based on retinex theory, 9th International Colloquium on Signal Processing and its Applications (CSPA), IEEE, 2013.

12. Rabeux Vincent, Journet N., Vialard A., and Domenger J.-P., Quality evaluation of ancient digitized documents for binarization prediction, 12th International Conference on Document Analysis and Recognition (ICDAR), IEEE, 2013.

13. Seki Minenobu, Asano E., Yasue T., and Nagayoshi H., Color Drop-Out Binarization Method for Document Images with Color Shift, 12th International Conference on Document Analysis and Recognition (ICDAR), IEEE, 2013.

14. Sehad Abdenour, Chibani Y., Cheriet M., and Yaddaden Y., Ancient degraded document image binarization based on texture features, 8th International Symposium on Image and Signal Processing and Analysis (ISPA), IEEE, 2013.

15. Nafchi Hossein Ziaei, Reza Farrahi Moghaddam, and Mohamed Cheriet, Application of Phase-Based Features and Denoising in Postprocessing and Binarization of Historical Document Images, 12th International Conference on Document Analysis and Recognition (ICDAR), IEEE, 2013.

16. Parker Jon, Frieder Ophir, and Gideon Frieder, Automatic Enhancement and Binarization of Degraded Document Images, 12th International Conference on Document Analysis and Recognition (ICDAR), IEEE, 2013.

17. Papavassiliou Vassilis, Simistira F., Katsouros V. and Carayannis G., A Morphology Based Approach for Binarization of Handwritten Documents, International Conference on Frontiers in Handwriting Recognition (ICFHR), IEEE, 2012.

18. Bolan Su, Shijian Lu, and Chew Lim Tan., A learning framework for degraded document image binarization using Markov random field, 21st International Conference on Pattern Recognition (ICPR), IEEE, 2012.

19. Patvardhan C., Verma A.K., and Vasantha Lakshmi C., Document image denoising and binarization using Curvelet transform for OCR applications, Nirma University International Conference on Engineering (NUICONE), IEEE, 2012.

20. Vavilis Sokratis, Ergina Kavallieratou, Roberto Paredes, and Kostas Sotiropoulos, A Hybrid Binarization Technique for Document Images, Learning Structure and Schemas from Documents, SCI 375, pp. 165-179, Springer-Verlag Berlin Heidelberg, 2011.

21. Yudong Zhang and Lenan Wu, Fast Document Image Binarization Based on an Improved Adaptive Otsus Method and Destination Word Accumulation, Journal of Computational Information Systems 7: 6 (2011) 1886-1892, June 2011.

22. Bolan Su, Shijian Lu and Chew Lim Tan, Combination of Document Image Binarization Techniques, ICDAR - International Conference on Document Analysis and Recognition, IEEE, 1520-5363/11, DOI:10.1109, 2011.

23. Soharab Hossain Shaikh, Asis Maiti and Nabendu Chaki, Image Binarization Using Iterative Partitioning: A Global Thresholding Approach, International Conference on Recent Trends in Information Systems, IEEE, 978-1-4577-0792-6/11, 2011.

24. Wassim Al-Khawand, Seifeddine Kadry, Riccardo Bozzo, and Khaled Smaili, Reading Skewed Images Without Image Rotation, British Journal of Mathematics \& Computer Science, ISSN: 2231-0851,Vol.: 4, Issue.: 7, 2014.

25. Wassim Al-Khawand, Seifeddine Kadry, Riccardo Bozzo, and Khaled Smaili, A Novel Skew Estimation Approach Based on Same Height Grouping, International Journal of Signal Processing, Image Processing and Pattern Recognition, Vol.7, No.3, pp.421-432, 2014. 